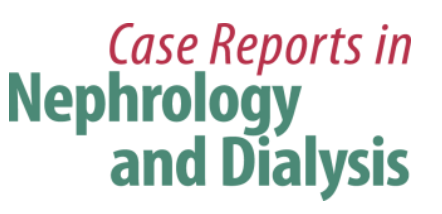

Case Rep Nephrol Dial 2016;6:101-105

\title{
A Unique Cause of Proteinuria in Pregnancy: Class II Lupus Nephritis with Concomitant Minimal Change Disease
}

\author{
Ryan Kunjal $^{\mathrm{a}} \quad$ Rabie Adam-Eldien $^{\mathrm{b}} \quad$ Raafat Makary $^{\mathrm{c}}$ Francois Jo-Hoy $^{\mathrm{a}}$ \\ Charles W. Heilig ${ }^{b}$ \\ ${ }^{a}$ Department of Internal Medicine, University of Florida College of Medicine, \\ Jacksonville, Fla., USA; ${ }^{b}$ Department of Nephrology, University of Florida College of \\ Medicine, Jacksonville, Fla., USA; 'Department of Pathology, University of Florida College \\ of Medicine, Jacksonville, Fla., USA
}

\section{Keywords}

Pregnancy · Systemic lupus erythematosus · Lupus nephritis · Minimal change disease

\begin{abstract}
We report the case of a 22-year-old African American female who presented to another facility for routine follow-up in the 34th week of pregnancy with lower extremity swelling and nephrotic-range proteinuria. Although she was normotensive, it was initially thought that she had preeclampsia. She was monitored carefully and delivery was induced at 37 weeks of gestation. She was transferred to our hospital, where she was diagnosed with systemic lupus erythematosus (SLE) based on clinical and laboratory criteria. Renal biopsy revealed a surprising finding of minimal change disease (MCD) concomitant with class II lupus nephritis (LN). She was managed with pulses and then tapering doses of steroid therapy with dramatic resolution of the nephrotic syndrome. This case demonstrates not only the rare de novo occurrence of SLE in pregnancy, but the unique finding of MCD coexisting with class II LN. We propose that altered T cell activity may be the link between these seemingly distinct entities.
\end{abstract}




\section{Case Reports in Nephrology and Dialysis}

\begin{tabular}{l|l}
\hline DOI: $10.1159 / 000448731$ & C 2016 The Author(s). Published by S. Karger AG, Basel
\end{tabular} www.karger.com/cnd

Kunjal et al.: A Unique Cause of Proteinuria in Pregnancy: Class II Lupus Nephritis with Concomitant Minimal Change Disease

\section{Introduction}

Systemic lupus erythematosus (SLE) commonly affects females of reproductive age and rarely occurs de novo in pregnancy $[1,2]$. Roughly $60 \%$ of SLE patients develop lupus nephritis (LN) with increased risk of neonatal and maternal morbidity [3]. It is also estimated that one third of patients with LN develop nephrotic-range proteinuria, which has only been rarely reported in class I and II LN as classified by the International Society of Nephrology/Renal Pathology Society (ISN/RPS) [4]. Herein we report a unique case of SLE diagnosed de novo in pregnancy complicated by concomitant minimal change disease (MCD) and class II LN on renal biopsy.

\section{Case Presentation}

A 22-year-old African American female was transferred to our hospital after delivery of her first live birth. Her medical history was significant for two prior spontaneous secondtrimester miscarriages. She was not on any medications and had a maternal history of SLE. She initially presented to another facility for a routine antenatal visit in her 34th week of gestation and at that time complained of pedal edema. Her urinalysis showed 4+ protein, but no white or red blood cells. Her 24-hour urine protein was $10.9 \mathrm{~g}$ and she was admitted with suspicion of preeclampsia. However, she remained normotensive and lacked features of HELLP syndrome (hemolytic anemia, elevated liver enzymes and low platelets) or neurological disturbances. Laboratory investigations revealed a normal uric acid level, but elevated serum creatinine $(1.4 \mathrm{mg} / \mathrm{dl})$, hypoalbuminemia $(1.6 \mathrm{~g} / \mathrm{dl})$, elevated triglycerides $(1,225 \mathrm{mg} / \mathrm{dl})$ and total cholesterol $(404 \mathrm{mg} / \mathrm{dl})$. Fetal growth was monitored and delivery was induced at 37 weeks of gestation.

Upon arrival at our hospital, the patient had no complaints apart from the progression of symmetric lower extremity swelling. Her physical examination was unremarkable except for an erythematous malar rash that she had not previously noticed and bilateral pedal edema. Repeat laboratory investigations were consistent with those done antepartum except for worsened creatinine at $2 \mathrm{mg} / \mathrm{dl}$. The spot urine protein to creatinine ratio was 7.6. Further evaluation of the nephrotic syndrome revealed speckled ANA (1:160 titer) as well as positive anti-DsDNA, anti-Sm and anti-RNP antibodies. C3 and C4 complement levels were normal. Tests for ANCA, SSA/SSB, RF, anti-CCP, Hep C Ab, Hep BsAg, and HIV were all negative. Antiphospholipid antibody screening was positive for anti-cardiolipin antibodies. Renal sonogram was unremarkable.

On postpartum day 8, renal biopsy of 30 glomeruli showed a mild increase in mesangial cellularity and matrix consistent with class II LN. There was no segmental or global sclerosis, endocapillary proliferation, or cellular or fibrous crescents. Immunofluorescence studies were positive for focal mesangial granular deposits of IgG, IgA and C1q, but no definitive IgM or C3 deposits were seen. No fibrin thrombi were identified. Surprisingly, on electron microscopy the glomerular basement membrane was mildly thickened up to $521 \mathrm{~nm}$ with patchy effacement of foot processes. Scant mesangial electron-dense deposits with focal increase in mesangial matrix as well as tubuloreticular inclusions in the glomerular capillary endothelium were also identified. Figure 1 and figure 2 demonstrate these findings.

Based on the clinical features, laboratory data and biopsy findings, a diagnosis of SLE with class II LN in addition to MCD was established. A detailed review of the patient's history and previous laboratory data did not raise any suspicion of indolent SLE prior to this pre- 


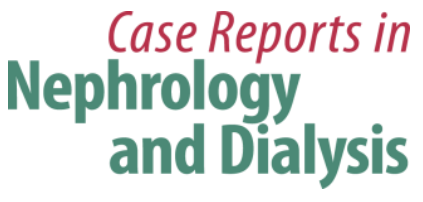

Case Rep Nephrol Dial 2016;6:101-105

DOI: 10.1159/000448731

C 2016 The Author(s). Published by S. Karger AG, Basel www.karger.com/cnd

Kunjal et al.: A Unique Cause of Proteinuria in Pregnancy: Class II Lupus Nephritis with Concomitant Minimal Change Disease

sentation. Treatment with methylprednisolone $1 \mathrm{~g}$ daily for 3 days followed by $1 \mathrm{mg} / \mathrm{kg}$ daily resulted in a dramatic improvement typical of MCD. Within 1 month, her creatinine returned to baseline and urinalysis showed only trace protein. Of note, repeat testing for anti-phospholipid antibodies at a 6-month follow-up visit was not consistent with antiphospholipid syndrome.

\section{Discussion}

SLE is diagnosed by means of criteria from the American College of Rheumatology. Our patient fulfilled four of these, including malar rash, positive ANA, presence of other autoantibodies and persistent proteinuria. Moreover, kidney biopsy revealed organized subcellular tubuloreticular inclusions in the endothelium, which are strongly associated with LN, albeit of higher classes than in our patient [5]. It is important to note that there have been only three previous reported cases of SLE occurring de novo in pregnancy [1, 2]. Our patient differed in that she presented in her third trimester, which posed a diagnostic challenge for two main reasons. Firstly, the more likely cause of proteinuria at that stage of pregnancy was preeclampsia. However, the absence of hypertension, hyperuricemia and features of HELLP syndrome made preeclampsia an unlikely etiology. Secondly, a renal biopsy could have revealed the underlying pathology, but it was not advised in late pregnancy due to the risk of complications [3]. As a result, her obstetrician opted for close monitoring and timely induction, thus allowing for a postpartum biopsy.

The risk of preterm delivery and fetal loss is higher in pregnant patients with SLE and further increased in those with LN [6]. Nonetheless, the occurrence of SLE in pregnancy may not simply be a coincidence. SLE is a Th2-mediated disease characterized by predominantly antibody-mediated immune responses. The immune system in pregnancy is ever evolving to preserve tolerance of the foreign paternal antigens carried by the fetus. As with SLE, there is a shift away from Th1 cell-mediated to Th2 antibody-mediated responses [4], which may be the unifying pathway between these otherwise seemingly distinct processes.

Another interesting aspect of this case was the concomitant finding of MCD with class II LN on renal biopsy. Nephrotic syndrome is mainly associated with class III, IV and V LN, whilst patients with class I and II LN have $<1 \mathrm{~g} / 24 \mathrm{~h}$ proteinuria [7]. In 1984, Abuelo et al. [8] reported the first case of MCD associated with SLE. Since then there have been approximately 38 more cases described in the literature $[9,10]$, but only 7 have involved class II LN [7, $11,12]$. Again, the coexistence of these two processes may be more than just chance. The pathogenesis of MCD is not yet fully understood, but factors derived from T cells that increase glomerular permeability have been reported culprits [13]. As mentioned before, altered $\mathrm{T}$ cell activity seems to be also critical to the pathogenesis of SLE and may be the link between the simultaneous occurrences of MCD in SLE. Additionally, there have been two previous case reports of biopsy-proven de novo MCD in pregnancy in the absence of SLE [14]. For similar reasons, the Th2 polarization driven by fetal tolerance may have been the impetus for the development of MCD in these patients. The interplay between these three processes - SLE, pregnancy and MCD - is fascinating, and further work is needed to elucidate the underlying pathophysiology.

Class II LN by itself does not generally warrant immunosuppressive therapy. Wong [10] noted that almost all cases of MCD in SLE were treated with steroids and were steroidsensitive, as expected for MCD. Had our patient presented earlier in pregnancy or with a higher class of LN, we would have been challenged to avoid the use of potentially teratogenic 
immunosuppressants to achieve disease remission. Instead, her response was rapid, with an outcome of a healthy newborn and control of her disease.

Perhaps MCD is an underdiagnosed part of the LN spectrum. The altered immune state of pregnancy may have precipitated not only MCD, but also SLE in our patient. As a consequence, the cause of her proteinuria was not initially obvious, and the renal biopsy was crucial in unmasking the underlying pathology. This case highlights the importance of thorough investigation of proteinuria in pregnancy and raises awareness of the occurrence of MCD in lower classes of LN.

\section{Statement of Ethics}

The authors have no ethical conflicts to disclose.

\section{Disclosure Statement}

The authors have no conflicts of interest or other support or financial disclosures.

\section{References}

1 Ergin RN: Pregnancy-associated systemic lupus erythematosus. Proc (Bayl Univ Med Cent) 2014;27: 221-222.

2 Patel T, Fenves A, Colbert G: The de novo diagnosis of systemic lupus erythematosus and lupus nephritis during pregnancy. Proc (Bayl Univ Med Cent) 2012;25:129-131.

-3 Stanhope TJ, White WM, Moder KG, Smyth A, Garovic VD: Obstetric nephrology: lupus and lupus nephritis in pregnancy. Clin J Am Soc Nephrol 2012;7:2089-2099.

- 4 Nam KH, Cha YJ, Kwon YE, Kim YL, Park KS, An SY, Lim BJ, Jeong HJ, Oh HJ, Yoo TH, Kang SW, Choi KH, Han SH: Minimal change disease in systemic lupus: another renal manifestation of lupus? Ewha Med J 2013;36:139-143.

5 Hala K: Tubulo-reticular inclusions in lupus nephritis: are they relevant? Saudi J Kidney Dis Transpl 2013;25:539-543.

-6 de Jesus GR, Mendoza-Pinto C, de Jesus NR, Dos Santos FC, Klumb EM, Carrasco MG, Levy RA: Understanding and managing pregnancy in patients with lupus. Autoimmune Dis 2015;2015:943490.

-7 Moysés-Neto M, Costa RS, Rodrigues FF, Vieira Neto OM, Reis MA, Louzada Júnior P, Romão EA, Dantas M: Minimal change disease: a variant of lupus nephritis. NDT Plus 2011;4:20-22.

8 Abuelo JG, Esparza AR, Garella S: Steroid-dependent nephritic syndrome in lupus nephritis. Arch Intern Med 1984;144:2411-2412.

- Kraft SW, Schwartz MM, Korbet SM, Lewis EJ: Glomerular podocytopathy in patients with systemic lupus erythematosus. J Am Soc Nephrol 2005;16:175-179.

10 Wong KW: A case of minimal change disease complicated by acute kidney injury in systemic lupus erythematosus. Saudi J Kidney Dis Transpl 2014;25:1308-1311.

11 Dube GK, Markowitz GS, Radhakrishnan J, Appel GB, D’Agati VD: Minimal change disease in systemic lupus erythematosus. Clin Nephrol 2002;57:120-126.

12 Stankeviciute N, Jao W, Bakir A, Lash JP: Mesangial lupus nephritis with associated nephrotic syndrome. J Am Soc Nephrol 1997;8:1199-1204.

$\checkmark 13$ Koyama A, Fujisaki M, Kobayashi M, Igarashi M, Narita M: A glomerular permeability factor produced by human T cell hybridomas. Kidney Int 1991;40:453-460.

$\$ 14$ Hamilton P, Myers J, Gillham J, Ayers G, Brown N, Venning M: Urinary protein selectivity in nephrotic syndrome and pregnancy: resurrection of a biomarker when renal biopsy is contraindicated. Clin Kidney J 2014;7:595-598. 


\section{Case Reports in \\ Nephrology and Dialysis}

Case Rep Nephrol Dial 2016;6:101-105 DOI: $10.1159 / 000448731$

(c) 2016 The Author(s). Published by S. Karger AG, Basel www.karger.com/cnd

Kunjal et al.: A Unique Cause of Proteinuria in Pregnancy: Class II Lupus Nephritis with Concomitant Minimal Change Disease

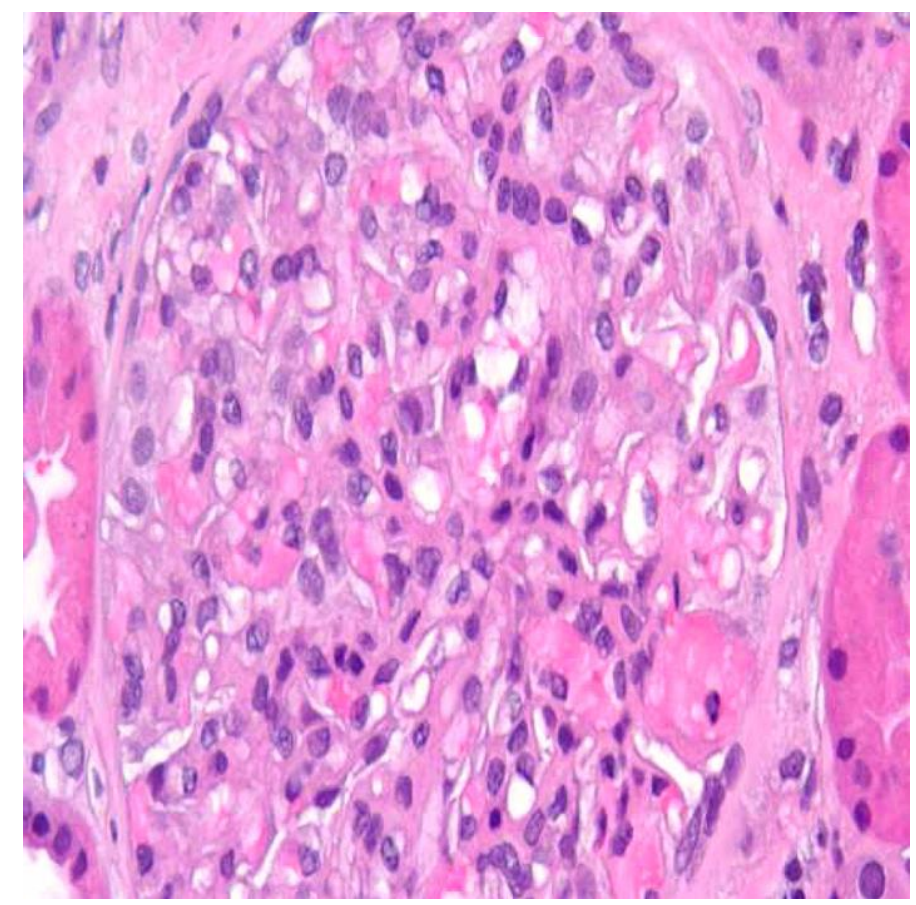

Fig. 1. Mild mesangial alteration on light microscopy (H\&E stain, $\times 40)$.

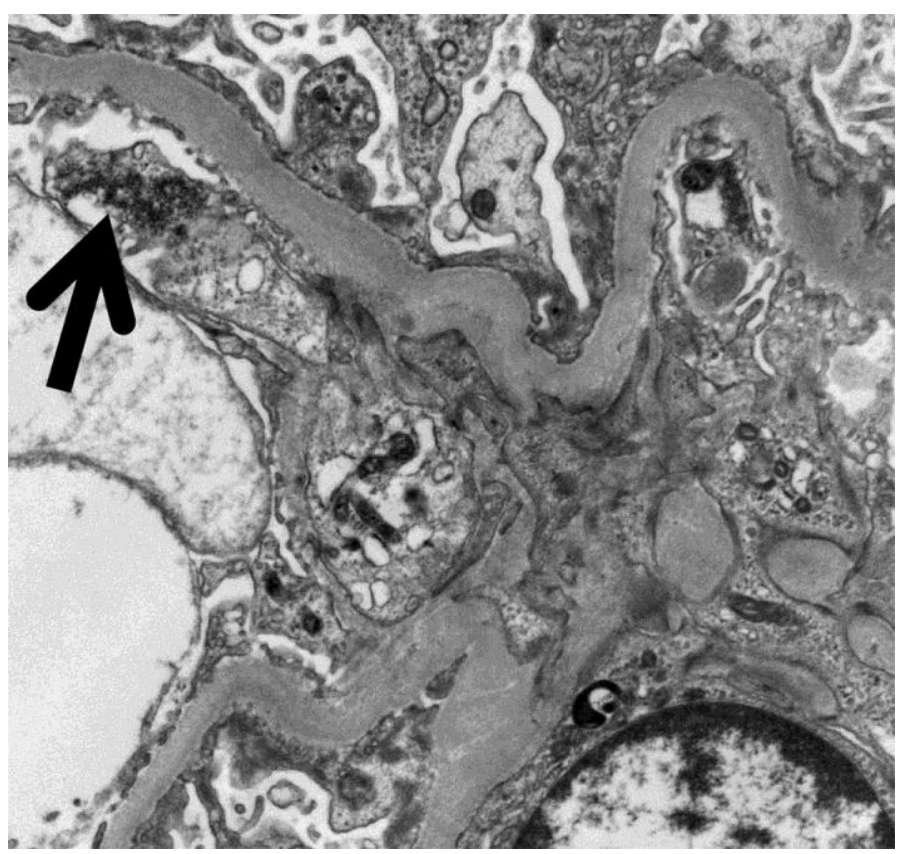

Fig. 2. Electron microscopic findings of endothelial tubuloreticular inclusions (arrow), slightly thickened glomerular basement membrane with foot process effacement and focal mesangial electron-dense deposits, but no evidence of subepithelial deposits $(\times 12,600)$. 